\title{
Numerical Simulation of Scour below Pipelines using Flexible Mesh Methods
}

\author{
J. M. Nunez Rattia, J. R. Percival, B. Yeager, S. Neethling, M. D. Piggott. \\ Department of Earth Science and Engineering \\ Imperial College London, SW7 2ZA, United Kingdom
}

\begin{abstract}
Evaluating bed morphological structure and evolution (specifically the scoured bed level) accurately using numerical models is critical for analyses of the stability of many marine structures. This paper discusses the performance of an implementation within Fluidity, an open source, general purpose, computational fluid dynamics (CFD) code, capable of handling arbitrary multi-scale unstructured tetrahedral meshes and including algorithms to perform dynamic anisotropic mesh adaptivity. The flexibility over mesh structure and resolution that these capabilities provide makes it potentially highly suitable for coupling the structural scale with larger scale ocean dynamics. In this very preliminary study the solver approach is demonstrated for an idealised scenario. Discontinuous Galerkin finite-element (DG-FEM) based discretisation methods have been used for the hydrodynamics and morphological calculations, and automatic mesh deformation has been utilised to account for bed evolution changes while preserving the validity and quality of the mesh. In future work, the solver will be used in three-dimensional impinging jet and other industrial and environmental scour studies.
\end{abstract}

\section{INTRODUCTION}

Historically, mathematical and computational models of scour below pipelines have been developed to describe the coupling between the hydrodynamic and morphological aspects of the scouring process. Advanced models calculate the flow around the pipe, solving the Navier-Stokes (N-S) equations numerically and in the cases of erodible bed, this also involves the numerical calculations of the bed morphology. Seabed geomorphologic models involve a sediment transport description to calculate scour/deposition processes. The key components of the sediment transport description are formulae for the bed-load and suspended sediment transport. One of the earliest full attempts to present a realistic dynamic description of the scour process at pipelines taking into account erosion and accretion caused by bed-load and suspended sediment was performed by Brørs (1999). In that approach a structured computational grid was used to model the scour under pipelines. With the development of unstructured grid models in computational fluid dynamics (CFD), methods for the more arbitrary resolution specification and updating of the computational mesh are available. The motivation is both to impart flexibility, e.g. to account for fluid-structure interactions, as well as to maximise computational efficiency, i.e. to minimise computational cost for a given accuracy, or to maximise accuracy for a given cost. Approaches for updating the mesh include techniques which perform local topological operations (e.g. sub-dividing elements, or changing mesh connectivity through the swapping of element faces and edges) to change the shape and size of mesh elements ( $h$-adaptivity or mesh optimisation), methods which continuously move the location of mesh vertices ( $r$-adaptivity or mesh redistribution/movement), and their combination in so-called $h r$-adaptive methods (Piggott et al. 2005).

This paper aims to describe a preliminary investigation of these issues. In particular, the current-induced local scour under a pipeline in live-bed conditions is considered. The model considered is built with the Fluidity framework (Piggott et al. 2008) and uses a single phase formulation with unstructured grids and discontinuous Galerkin (DG) finite element discretisation schemes for the velocity and pressure fields and a control volume $(\mathrm{CV})$ discretisation for the sediment concentration field. Local boundary adjustment of the seabed nodes provides a bed grid motion velocity field and an unstructured mesh smoothing technique (i.e. Laplace elliptic smoothing) is used to evolve the entire computational mesh in response. Mao's (1986) experimental setup has been used as a motivation for the idealised test case considered, and a demonstration of the capabilities of the developed model in the simulation of tunnel erosion and its subsequent 
stages.

\section{NUMERICAL MODEL}

\subsection{Model Setup}

This section concentrates on the mathematical formulation for the flow field model and the scour model which includes the sediment transport equations.

\subsubsection{Flow Model}

The motion of fluid can be described by the incompressible Navier-Stokes (N-S) equations, which include the continuity and the momentum equations,

$$
\left\{\begin{array}{l}
\nabla \cdot \boldsymbol{u}=0 \\
\frac{\partial \boldsymbol{u}}{\partial t}+\boldsymbol{u} \cdot \nabla \boldsymbol{u}=-\nabla \frac{p}{\rho_{0}}+\nu \nabla^{2} \boldsymbol{u}-\frac{\rho}{\rho_{0}} g \hat{k}
\end{array}\right.
$$

where $\boldsymbol{u}$ is the flow velocity field, $p$ is the pressure field, $g$ is the gravitational force (under the absence of any other forcing term) in a planar reference frame when gravity points in the negative vertical direction $\hat{k}=(0,0,1)^{T}, \rho_{0}$ is a reference water density (Boussinesq approximation, $\left.\left|\rho-\rho_{0}\right| \ll \rho_{0}\right)$ and $\nu$ is the kinematic fluid viscosity.

Based on the advancements in CFD algorithms, the N-S equations can be solved with different approaches. Also, the modification in flow pattern caused by the interaction of structures (e.g. pipelines) with the flow produces turbulence. Consequentially this affects the sediment transport in the channel. In order to accurately calculate local scour, normally the modelling of high Reynolds flow regimes are required, and ideally this involves solving the NavierStokes equations without averaging or approximation other than numerical simulation through Direct $\mathrm{Nu}$ merical Simulation (DNS). The results of DNS contain very detailed information about the flow and is more than is required for engineering purposes, as well as being computationally expensive.

Previous literature in regards to the simulation of local scour problems, in particular the scouring near horizontal pipes, have discussed turbulence modelling approaches, where velocities and pressure quantities may be decomposed into averaged (mean flow) and fluctuating (turbulent) components such as in Reynolds-Average Navier-Stokes (RANS) equations (e.g. k- $\epsilon$ or k- $\omega$ ) and Large Eddy Simulations (LES) (e.g. Smagorinsky sub-grid scale (SGS) models), which are generally less costly than DNS for small-scale flows with high Reynolds number and too complex geometry.

Fluidity (2015), an open source, general purpose computational fluid dynamics FEM code developed at Imperial College London (Piggott et al. 2008), has the capabilities of solving the N-S equations with sufficiently fine meshes (adaptive remeshing) and sufficiently small time steps (adaptive time stepping) to resolve with at great resolution the eddies and fluctuations in small-scale turbulence models (Parkinson et al. 2014). Although this is theoretically possible, for many engineering applications it is not always necessary to resolve all details of the turbulence fluctuations in a simulation, and some form of parametrisation is required. In this study no turbulence closure scheme was employed given that the purpose of this initial investigation was to tackle the bed scour modelling. The incompressible $\mathrm{N}-\mathrm{S}$ equations are resolved at the spatial grid-scale generated level sufficiently to capture the vortex shedding due to unsteady flow moving past an obstruction (i.e. pipeline). Downwind of the obstruction are regions of lower pressure which causes the deflected fluid to recirculate or form vortices.

\subsubsection{Bed Change Model}

Simulations of sediment transport coupled with bed evolution hereafter, the Exner equation are commonly conducted under the discretisation of a particular form of the general bed level change equation (Paola \& Voller 2005),

$$
\frac{\partial \eta}{\partial t}=-\frac{1}{1-n}\left[\nabla \cdot\left(\boldsymbol{q}_{b}+\boldsymbol{q}_{s}\right)-\frac{\partial}{\partial t} \int_{\eta}^{\eta+H} \boldsymbol{c} d z\right] .
$$

The Exner equation results from the conservation of mass and therefore from first principles. It states that the net balance between the gain and loss of mass (i.e. sediment) in a certain control volume results in a change of sediment height $\eta$ considering a porosity $(n)$ of the deposited sediment in the bed layer here assumed to be 1 as per compacted sediment. Assuming that the sediment concentration is steady and the suspended load can be expressed using explicit empirical descriptions, the last term in the previous equation, which represents the temporal change in the volume of sediment in the water column of height, $H$ (i.e. storage term) together with the transport vector of suspended sediment over the vertical direction (i.e. $q_{s}$ ), can be replaced with the entrainment rate,

$E=\Gamma \frac{\partial c}{\partial x_{z}}$

and the deposition rate,

$D=w_{s} c_{0}$

and therefore the Exner model adopts the simpler form of,

$\frac{\partial \eta}{\partial t}=-\frac{1}{1-n}\left(\nabla_{\text {horiz }} \cdot \boldsymbol{q}_{b}-D-E\right) ;$ 
where,

$\nabla_{\text {horiz }}=\left(\frac{\partial}{\partial x}, \frac{\partial}{\partial y}, 0\right) ;$

is the horizontal divergence of the bed-load flux.

The deposition rate $D$ is set equal to the sinking velocity of the sediment particles, denoted $w_{s}$, multiplied by the near-the-bed concentration $\left(c_{0}\right)$, and the entrainment rate $E$ is expressed in terms of the local diffusivity (or the diffusion coefficient, $\Gamma$ ) and the vertical concentration gradient. The value of $\Gamma$ is commonly assumed to be equal to the eddy viscosity (i.e. a turbulent Schmidt number of 1). Many empirical models are available in the literature for estimating the entrainment rate of sediment into suspension. Garcia \& Parker (1991) performed detailed comparison of formulae and validated these against laboratory results, and produced an improved formula for sediment re-entrainment, which was chosen to use in the current study.

It is worthwhile noting that the rate of bed change as expressed in (5) depends on scalar quantities on the right hand side (i.e. $D$ and $E$ as deposition and erosion/entrainment), which results in a big simplification for the suspended sediment transport given that the numerical integration over the vertical direction is not needed, this makes it easy to evaluate the rate of bed level change for complex geometries or unstructured meshes.

Although the dominant sediment transport mode (small Rouse number) under slightly severe environmental conditions such as flow in channels is the suspended load (normally omitted in previous numerical models), which assumes the sediments travel above the bed layer, a different mechanism known as the bed-load, which assumes those particles are transported along the bed, is commonly combined into the holistic bed change model. The bed-load are commonly described using empirical or semi-empirical equations. They are often determined by the local bed shear stress and sediment characteristics. In this present study the Engelund \& Fredsøe (1976) equations were selected for the calculation of the bed-load flux,

$\Phi=18.74\left(\theta-\theta_{c}\right)\left(|\theta|^{\frac{1}{2}}-0.7\left|\theta_{c}\right|^{\frac{1}{2}}\right) ;$

where the non-dimensional parameters are the Einstein number,

$\Phi=\frac{\boldsymbol{q}_{b}}{R g d^{\frac{1}{2}} d} ;$

associated with the gravity acceleration of sediment in water $(R g)$, the sediment diameter $\left(d_{50}\right)$, the bedload sediment transport rate per unit width $\left(q_{b}\right)$, and the Shields number,

$\theta=\frac{\boldsymbol{\tau}_{b}}{\rho R g d}$ associated to the sediment diameter, the submerged sediment density $(\rho R)$, and the bed shear stress $\left(\tau_{b}\right)$. The critical Shields number $\left(\theta_{c}\right)$ is assumed to be zero, which is a conservative assumption for sandy bed layers (Graf 1998). Interesting corrections to the critical Shields parameter have been considered to account for non-planar geometries such as rocky or gravel seabed (Brørs 1999). In the case of sandy areas, $\theta_{c}$ can be neglected and the use of simpler models which do not take the incipient motion theory into account can be satisfying. For that reason, in this study no local slope correction was added into the bed shear stress calculation to account for the critical shear stress reduction at incipient motion on a sloping bed.

The bed shear stress and shear velocity are used to calculate the scour and deposition in the transport field. In complex flow fields, it is extremely difficult to calculate the correct value. Some bed shear stress formulations are available in the literature (e.g. quadratic stress law, friction based) such as the wall function calculation where the bed shear stress is calculated from the logarithmic relation between the shear velocity and the velocity change with height (Graf 1998). Although this is one of the most commonly used formulations in hydraulic and coastal engineering, the current study employs a more straightforward calculation based on the velocity field gradient,

$\boldsymbol{\tau}_{b_{i j}}=\nu\left(\frac{\partial u_{i}}{\partial x_{j}}+\frac{\partial u_{j}}{\partial x_{i}}\right)$.

\subsection{Problem Domain and Boundary Conditions}

In this study of current-induced scour simulation around a pipeline, a two-dimensional (2D) initially rectangular flow domain is considered, as shown in Figure 1. The domain (where D is the pipe diameter) is $30 \mathrm{D}$ long and $4 \mathrm{D}$ high. The pipe centre was located at 10D from the inlet boundary and the bottom of the pipe was positioned very close to the flat seabed with a gap (e) below the pipe to account for the onset of the scour stage. The gap between the bottom of the pipe and the top of the seabed was assumed initially to be $0.05 \mathrm{D}$.

The simulated flows are steady pressure-driven water tunnel (rigid lid) flows in the domain height. At the inlet (left-hand side) boundary, a logarithmic velocity profile was applied (common in channel geometries). At the outlet boundary the pressure is set to zero. At the solid surfaces (seabed and pipeline) a no slip condition, $\boldsymbol{u}=0$, is set for the velocity field. In regards to the sediment boundary conditions, at the inlet the suspended sediment concentration $c$ is set to zero, which corresponds to clear water coming into the domain. For the solid walls, the flux of sediment through these surfaces is zero. At the sandy bed, the suspended sediment flux is specified as the net upward normal flux such that,

$\boldsymbol{q}_{s} \cdot \boldsymbol{n}=E-D$. 


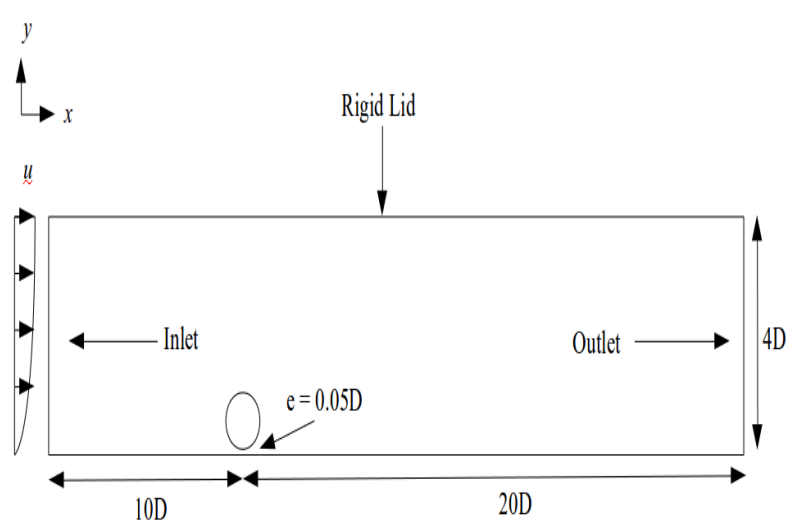

Figure 1: Sketch of physical domain where, D is the diameter of the circular pipe and $e$ is the gap between the pipe and the bottom boundary or seabed.

\subsection{Discretisation and Numerical Solution Procedure}

Equations (1) have been spatially discretised using a $\mathrm{P} 1_{D G} \mathrm{P} 2$ (following the nomenclature of Fluidity) finite element pair, in which velocity varies linearly across elements and is discontinuous between elements, and pressure has a quadratic variation across elements and is continuous between elements (Cotter et al. 2009). This is highly recommended for ocean applications of Fluidity for stability plus accuracy reasons and given that the mass matrix can be inverted locally, so it does not have to be lumped hence saving computational time. Some of the advantages of choosing discontinuous elements (as in the case of the velocity field) are having both the geometric flexibility and high-order accuracy offered by Finite Element Method (FEM) together with the local statement on the residual represented as numerical fluxes (e.g. upwind flux scheme chosen for the current study) across the element facets in the fashion of the Finite Volume Method (FVM).

Time discretisation for the N-S equations is via an operator splitting method, in which the convective term is solved explicitly with a subcycled timestep chosen such that the maximum CFL number for the system is below 0.2 and a slope limiting method is applied to the between element fluxes to damp numerical oscillations. The diffusive term is solved using a slighty implicit theta method timestepping and a global timestep chosen so that the maximum CFL number is below 10 .

The concentration field $(c)$ in the suspended sediment load is calculated by resolving the standard convection-diffusion equation,

$\frac{\partial \boldsymbol{c}}{\partial t}+\nabla \cdot \boldsymbol{c}\left(\boldsymbol{u}-w_{s} \hat{k}\right)=\nabla \cdot(\Gamma \nabla \boldsymbol{c})$

with the inclusion of the settling velocity of sediment $\left(w_{s}\right)$ in the downwards direction. The settling velocity normally decreases as the concentration of sediment increases, with this effect being taken into account by calculating a hindered sinking velocity,

$w_{s i}=w_{s 0}(1-c)^{2.39} ;$

from the unhindered settling velocity, $w_{s 0}$. The numerical treatment for this transport equation is done in a similar fashion to the momentum equations for the fluid flow. Due to the mass conservation nature of the sediment transport the numerical solution for the concentration field was achieved through a control volume discretisation which can be thought of being as the lowest order discontinuous Galerkin method $\left(\mathrm{P} 0_{D G}\right)$ using a vertex-centred scheme for the advection/diffusion equation.

Control Volumes (CV) handle the equations in a conservative form guaranteeing that the discrete approximations of the conserved quantities are preserved at the same face of adjacent volumes (conservativeness) and also in order to ensure boundedness (requirement for convergent iterative solution normally achieved if all coefficients from the discretisation have the same sign) for the chosen interpolation scheme in the facet values, a face value limiter (for this study a Sweby slope limiter (Sweby 1984)) is normally applied to compensate for unstable advective fluxes and maintain stability. A Crank-Nicolson timestepping procedure is used.

The evolving bed is represented by updating the position of the nodes in the bottom boundary at each time-step. The flow field model is first calculated by solving the Navier-Stokes equation. Then the obtained flow parameters are employed to solve the bedload and suspended load formulae allowing a calculation of a propagation velocity (i.e. grid velocity) in the water-sediment interface. The velocity of the boundary is determined by the mechanism of sedimentation given by the one-dimensional Exner equation in a two-dimensional flow domain (i.e. just considering the divergence of the bed-load flux in the $x$ direction).

The terms on the RHS of (5) are evaluated in the vertical direction to the sediment surface (i.e. bed level changes in the vertices on the deforming bed projected in the vertical axis). This is an assumption for the solution of the Exner equation given that, in reality, the bed predominantly changes along its normal direction, when sediment is eroded or deposited. The bed elevation change is due to the horizontal divergence of the bed-load sediment transport flux (which is easily obtained from (7) using the shear stress from the flow solver) together with erosion and deposition of sediment on the bed boundary.

It should be mentioned that it is a common practice in computational morphodynamics to use a morphological acceleration factor to decrease computational time by defining a time-step which is much longer than the flow time-step (perhaps 100 to 1000 times). This idea raises a concern in terms of mass conservation. The sediment in suspension presents a temporal lag which is not corrected if an acceleration factor is 
used and could lead to conservation errors. As mentioned before in this study the bed morphology model is represented by explicitly evolving the mesh points directly at each time-step.

The moving mesh method used to adjust interior node locations in this work in response to bed deformation is termed Laplacian smoothing and is achieved through the solution of an elliptic PDE. We define a computational domain (here taken to be the same as the initial physical domain geometry as per Figure 1p with coordinate vector $(\xi, \eta)$ and map it to the time-evolving physical domain with coordinate vector $(x, y)$ where the underlying physical solutions (i.e. pressure and velocities) are computed.

The mapping $(\xi, \eta) \rightarrow(x, y)$ is defined as the solution of the system of PDEs,

$$
\nabla^{2} x=x_{\xi \xi}+x_{\eta \eta}=0 ; \quad \nabla^{2} y=y_{\xi \xi}+y_{\eta \eta}=0 ;
$$

where the Laplacian operator is applied in the computational domain and with boundary conditions given by the current position of the physical domain boundaries. Solutions to this problem (and hence the mapping) are generally very smooth leading to smooth contours where the interior grid points are relocated in response to boundary deformation. The equations are solved at each time step with a degree 1 continuous Galerkin finite element method, implemented using the FEniCS/DOLFIN finite element software (Alnæs et al. 2015, Logg and Wells 2010).

\section{NUMERICAL SIMULATION AND RESULTS}

The numerical simulation was carried out against the laboratory experimental inputs from Mao's experiments (1986). In the experiment a hydraulically smooth pipe with a diameter of $0.1 \mathrm{~m}$ was initially placed over the bed (sand layer), which had median particle diameter of about $0.36 \mathrm{~mm}\left(d_{50}\right)$ and a mean flow velocity of $0.5 \mathrm{~m} / \mathrm{s}$ corresponding to a Shields parameter of $\theta=0.098$.

In the present study, the early stages of scour was simulated by assuming a flat bed profile under the pipe with a small gap $(e \ll D)$ between the bottom of the pipe and the seabed. This was done in order to skip the onset of scour underneath the pipeline. When the gradient in the pressure difference between the upstream and the downstream of the pipe (seepage flow) reached a critical value for seepage failure, tunnel erosion can be initiated and the current velocity in the gap increases rapidly (Sumer \& Fredsøe 2002). This onset phase was omitted in this study because the physics of the seepage flow within the porous soil underneath the pipeline was not included. Instead we start with an initial state (i.e. tunnel erosion) where for an instant the gap between the pipe and the bed remains small (i.e. 5 $\mathrm{mm}$ ) and during this stage, water is diverted to the gap leading to increased velocities in the gap and resulting in very large shear stresses on the bed just below the pipeline. In the initial configuration the mesh was refined in between the gap with sufficient extra layers of elements between the bottom of the pipe and the bottom boundary so more vertices could be moved during the mesh deformation and smoothing process.

In the previous numerical models (structured mesh) for simulations of the local scour below pipelines, an artificial sinusoidal shape hole beneath the pipeline was introduced to avoid complete remeshing during the calculation (e.g. Brørs (1999) and Liang, Cheng, \& Li (2005)). It has been observed and also tested in the current study that the introduction of the initial scour hole has negligible effect on the subsequent predicted scour profiles.

In the numerical simulation, the flume used for the experiments was assumed to be infinitely wide, so that a two-dimensional approach is possible. The most important input parameter assumptions are:

- unhindered settling particle velocity; $w_{s 0}=0.01$ $m / s$, and;

- submerged specific gravity of sediment; $\mathrm{R}=1.65$ for a typical situation, such as quartz-rich sediment.

With a Shields parameter, $\theta=0.098$ from the laboratory experiments, during the early stage of the scour the erosion rate was very high due to the tunnel erosion, so that a scour depth reached $4 \mathrm{~cm}$ after 1.5 minutes. The equilibrium stage was reached after 200 minutes with a maximum scour depth of $8 \mathrm{~cm}$. Due to the computational cost required to run the simulation for longer times, results were obtained during the early stages of the tunnel erosion scour development (approximately 2 minutes).

Figure 2 illustrates the the velocity field and bed profiles around the pipeline at different times $(0.1$ min, $1 \mathrm{~min}, 1.5 \mathrm{~min}$ and $2 \mathrm{~min}$ ). Some of these stages and scour development profiles are qualitatively comparable to bed profiles from Jensen et al. (1990).

It is seen at the very early stages of scour (profile $I$ ), when the scour depth is very small, that the water flows in the gap forming a jet scour pushing out a substantial amount of sand and, depositing the sand just behind the pipe. In this profile no downstream vortex shedding can yet be traced.

In profile $I I$ the tunnel erosion has now created a scour hole underneath the pipe and the bed change slows down as the gap becomes larger due to scour. This following stage is called lee-wake erosion where the vortex shedding controls the scour. Experimentally it was observed that during this phase more scour will occur at the downstream side of the pipe than at the upstream, removing the dunes of deposited sediment from the tunnel erosion. Our model is able to predict a faster upstream erosion and also a typical ripple regime in the downstream section although it currently seems to under-predict the dune formation behind the pipeline. These disparities were also reported in previous numerical simulations by Liang, Cheng, \& Li (2005) using a Smagorinsky subgrid scale (SGS) turbulunce model and the results were at- 


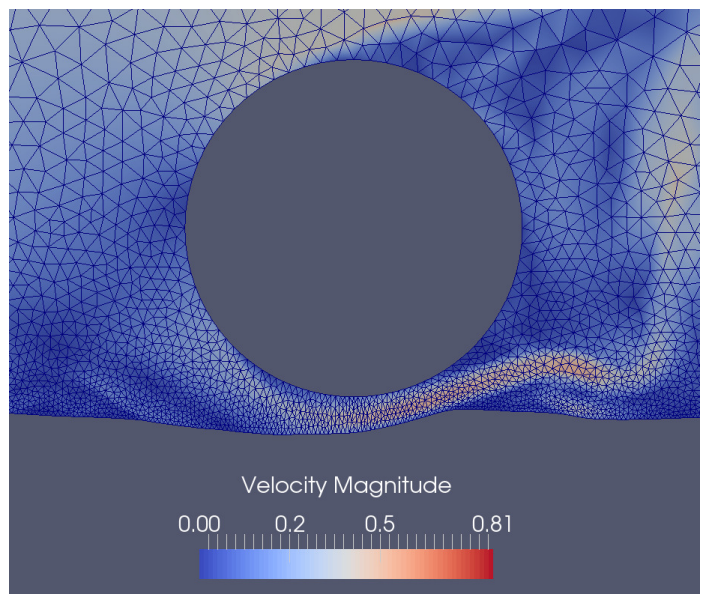

(a) $0.1 \mathrm{~min}$. Profile I

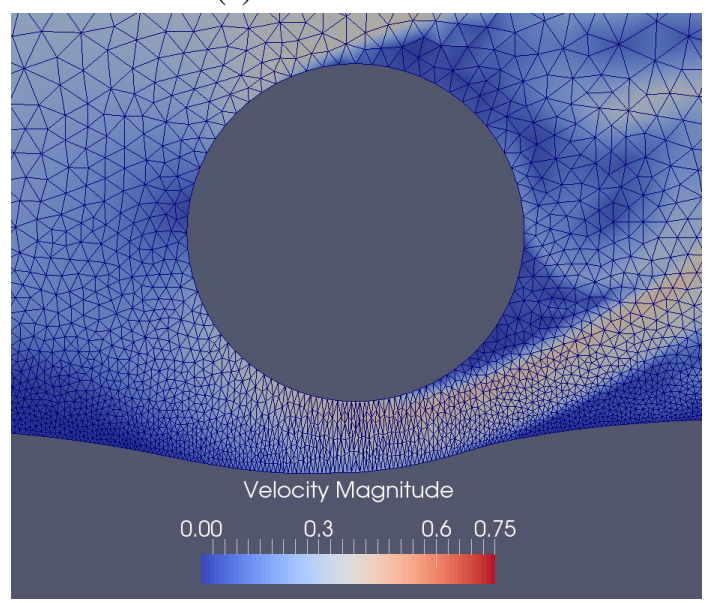

(b) 1 min. Profile II

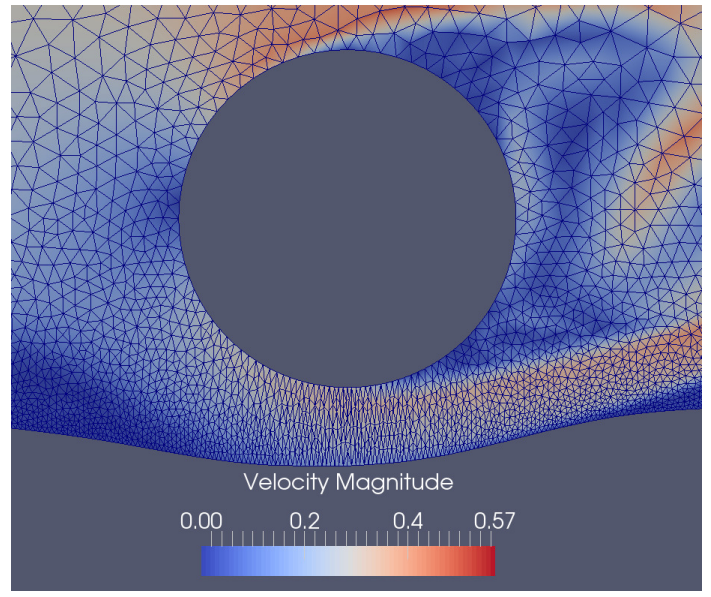

(c) $1.5 \mathrm{~min}$. Profile III

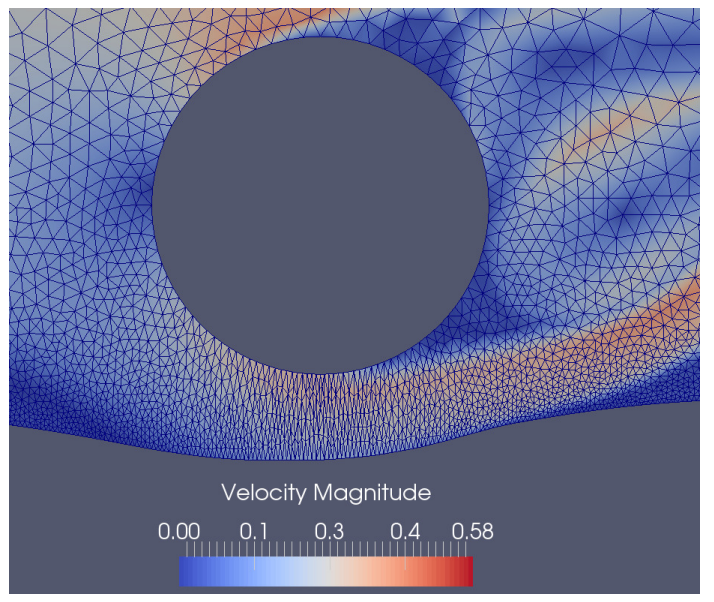

(d) 2 min. Profile IV

Figure 2: Bed profile visualisations and velocities magnitudes at different times. tributed to overestimation of the interaction between the vortices shed from the pipe and the bed for which a k- $\epsilon$ model was recommended for better predictions.

As the scour depth increases (profile III) and the gap becomes bigger the eddies became weaker and finally vanished. Therefore the mean gap velocity and bed shear stress remain approximately constant for further increase of scour depth and no major changes in the bed profiles are observed, the scour process reaches a steady state of equilibrium stage (profile $I V)$.

Also, it can be observed (as shown in Figure 3) that the adaptive mesh smoothing process is helping to avoid an excessive stretching of the bottom elements which otherwise will affect the mesh quality impacting the convergence rate and possibly the solution error.

Sometimes, bed slope instabilities often occur in the bed elevation resulting in unrealistic scour profiles given the complex nature of the sediment transport equations and the interaction with the flow field. These instabilities occur when the predicted slope of the scour hole exceeds the geotechnical stability of the sediment particles (normally taken as the angle of repose, $\Phi)$. Some numerical algorithms have been proposed to overcome these difficulties like for example, a sediment/sand sliding model that artificially prevents the local boundary slopes to exceed the angle of repose or maximum friction angle.

At this current stage of the study, at the boundary nodes further smoothing during the adaptive mesh movement was applied with the implementation of a low-pass filter in order to average out rapid changes in the grid velocity provoking large bed slopes. This potentially is adding some inaccuracy in the resolved bed profile. However, it is expected that the future model employing a sediment slide technique will improve the capability of the present model.

Figure 4 shows the progression of the bed profiles for this particular study at an different time measurements. The generated curves indicate the present model provides good estimations of the bed shapes (concave profiles) during its evolution.

\section{CONCLUSIONS}

This paper presents ongoing work on the verification of a novel two-dimensional discontinuous finiteelement model with adaptive moving mesh techniques for the simulation of scouring underneath subsea pipelines in steady currents. The inclusion of $r$ adaptivity or moving mesh strategies such as Laplacian smoothing which involves the repositioning of the nodes of the mesh, permits the adjustment of the mesh in an efficient way for simulations with evolving boundaries like in the case of seabed scour underneath pipelines. Although the model results are stable for the experimental conditions considered, some differences, specifically in the scour depth were noticed 


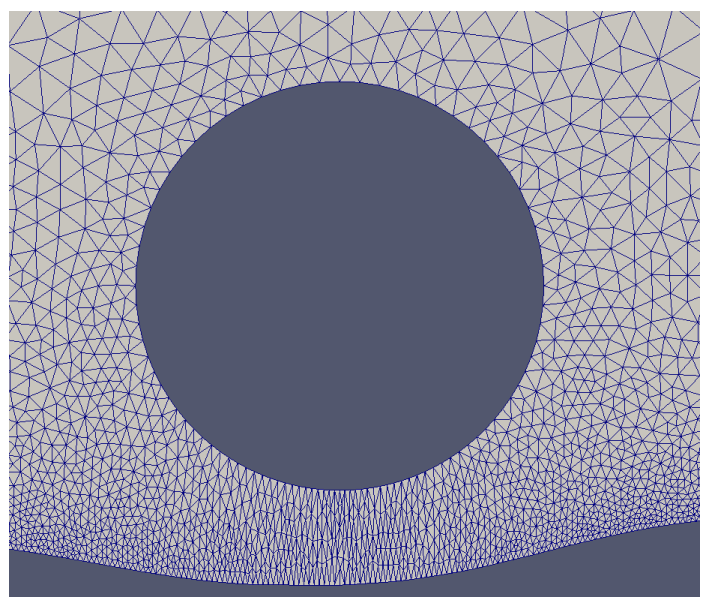

(a) Mesh with Laplacian smoothing

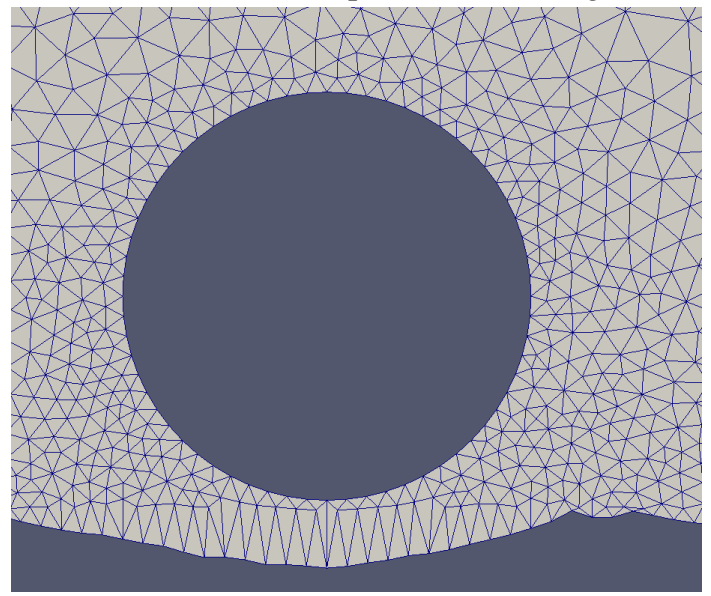

(b) Mesh without Laplacian smoothing

Figure 3: Comparison between physical meshes with and without Laplacian smoothing at $\mathrm{t}=1.5 \mathrm{~min}$.

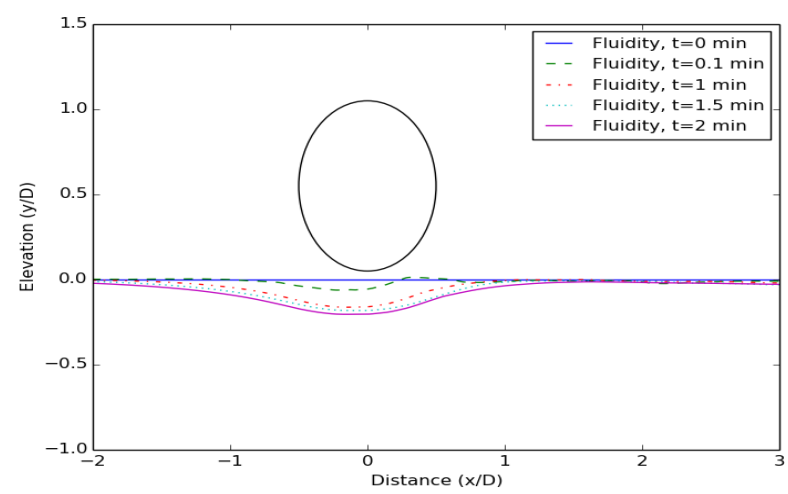

Figure 4: Bed evolution at different times for the current numerical model using experimental data from case $\theta=0.098$. when compared with previous numerical predictions and experimental results.

Among the open issues to be considered in future development of this model is the choice of a turbulence model. In this current work the possible effects of how the turbulent energy can be affected by the presence of sediment are not yet included. This seems to be a critical aspect in the interaction between the vortex shedding from the pipe and the bed, hence the underprediction of the scour profiles at the early stage of the scour. In addition, the treatment of the flow near the boundaries is of extreme importance, generally speaking two approaches can be distinguished to tackle this for $\mathrm{k}-\epsilon$ turbulence models, with wallfunction meshes that will bridge the gap in the boundary layer with a wall function approach, and with the application of fine meshes where the appropriate low-Reynolds-number boundary conditions will be applied. The last strategy could be potentially benefited using adaptive gridding techniques such as $h$ adaptivity (Piggott et al. 2005) techniques or mesh refinement strategies in order to impart higher resolution in the model. Another option for the turbulence modelling may be to consider large eddy simulations (LES) methods based on space-filtered equations where large eddies are explicitly calculated and the effect of small eddies are modelled with a sub-grid parametrisation similar to the eddy-viscosity models.

Also, future work is expecting to extend the bedload sediment transport formulas to include (vectorially) the gravitational influence on large bed slopes. The effect of a sloping bottom is to increase the bedload transport rate in the downslope direction, and to reduce it in the upslope bed-load direction. A correction factor can be applied to both the magnitude and direction of the solid transport rate, before solving the bed evolution equation. In addition, a sediment slide model algorithm to control the bed-load flux when the slope angle exceeds the angle of repose will be included.

A fully robust sediment transport and morphodynamic model is expected to be used in applications that include the scouring behaviour of a threedimensional impinging jets (Robinson et al. 2015), which will help answer questions about marine hazard processes in polar regions related to river flooding of fast ice and subsequent strudel scour formation. When the flow involves jets, separation or moderate swirl, more rigorous techniques are required to capture the effect of turbulence, e.g. in the spreading of a round jet.

Multiphase models considering flow-sediment interactions will also be explored with the application of meshless methods for comparison purposes.

\section{ACKNOWLEDGEMENT}

The authors would like to acknowledge the work of the Fluidity development team and also the financial 


\section{REFERENCES}

Alnæs, M. S., J. Blechta, J. Hake, A. Johansson, B. Kehlet, A. Logg, C. Richardson, J. Ring, M. E. Rognes, \& G. N. Wells (2015). The fenics project version 1.5. Archive of $\mathrm{Nu}$ merical Software. 3(100), 9-23.

Applied Modelling and Computational Group (2015). Fluidity Manual Version 4.1 branch 4112 Release. Department of Earth Science and Engineering, Imperial College London. DOI: $10.6084 / \mathrm{m} 9$. figshare.1387713.

Brørs, B. (1999). Numerical modeling of flow and scour at pipelines. J. Hydraul. Eng. 125(5), 511-523.

Cotter, C. J., D. A. Ham, \& C. C. Pain (2009). A mixed discontinuous/continuous finite element pair for shallow-water ocean modelling. Ocean Modelling. 26(12), 86-90.

Engelund, F. \& J. Fredsøe (1976). A sediment transport model for straight alluvial channels. Nordic Hydrology. 125(5), 293-306.

Garcia, M. \& G. Parker (1991). Entrainment of bed sediment into suspension. J. Hydraul. Eng. 117, 414-435.

Graf, W. (1998). Fluvial Hydraulics: Flow and Transport Processes in Channels of Simple Geometry. McGraw-Hill. ISBN: 978-04719777148.

Jensen, B., B. Summer, H. Jensen, \& J. Fredsøe (1990). Flow around and forces on a pipeline near a scoured bed in steady current. Offshore Mechanics and Arctic Engineering (OMAE). 112, 206-213.

Liang, D., L. Cheng, \& F. Li (2005). Numerical modeling of flow and scour below a pipeline in currents part II: Scour simulation. J. Coastal Engineering. 52, 43-62.

Logg, A. \& G. N. Wells (2010). Dolfin: Automated finite element computing. ACM Transactions on Mathematical Software. 37(2).

Paola, C. \& V. R. Voller (2005). A generalized exner equation for sediment mass balance. J. Geophys. Res. 110(F04014).

Parkinson, S., J. Hill, M. Piggott, \& P. Allison (2014). Direct numerical simulations of particle-laden density currents with adaptive, discontinuous finite elements. J. Geosci. Model. Dev. 7, 1945-1960.

Piggott, M., G. Gorman, C. Pain, P. Allison, A. Candy, B. Martin, \& M. Wells (2008). A new computational framework for multi-scale ocean modelling based on adapting unstructured meshes. Int. J. for Numerical Methods in Fluids. 56, 10031015.

Piggott, M. D., C. C. Pain, G. J. Gorman, P. W. Power, \& A. J. H. Goddard (2005). $h, r$, and $h r$ adaptivity with applications in numerical ocean modelling. Ocean Model. 10, 95-113.

Robinson, D., M. Wood, M. Piggott, \& G. Gorman (2015). CFD modelling of marine discharge mixing and dispersion. J. of Applied Water Engineering and Research. In press. DOI: 10.1080/23249676.2015.1105157.

Sumer, B. M. \& J. Fredsøe (2002). The Mechanics of Scour in the Marine Environment. World Scientific. ISBN: 9789810249304

Sweby, P. K. (1984). High-resolution schemes using flux limiters for hyperbolic conservation-laws. SIAM J. on Numerical Analysis. 21, 995-1011. 